\title{
Detection of Zeptojoule Microwave Pulses Using Electrothermal Feedback in Proximity-Induced Josephson Junctions
}

\author{
J. Govenius," R. E. Lake, K. Y. Tan, and M. Möttönen \\ QCD Labs, COMP Centre of Excellence, Department of Applied Physics, Aalto University, \\ P.O. Box 13500, FIN-00076 Aalto, Finland
}

(Received 22 December 2015; revised manuscript received 4 May 2016; published 15 July 2016)

\begin{abstract}
We experimentally investigate and utilize electrothermal feedback in a microwave nanobolometer based on a normal-metal $\left(\mathrm{Au}_{x} \mathrm{Pd}_{1-x}\right)$ nanowire with proximity-induced superconductivity. The feedback couples the temperature and the electrical degrees of freedom in the nanowire, which both absorbs the incoming microwave radiation, and transduces the temperature change into a radio-frequency electrical signal. We tune the feedback in situ and access both positive and negative feedback regimes with rich nonlinear dynamics. In particular, strong positive feedback leads to the emergence of two metastable electron temperature states in the millikelvin range. We use these states for efficient threshold detection of coherent $8.4 \mathrm{GHz}$ microwave pulses containing approximately 200 photons on average, corresponding to $1.1 \times 10^{-21} \mathrm{~J} \approx 7.0 \mathrm{meV}$ of energy.
\end{abstract}

DOI: 10.1103/PhysRevLett.117.030802

Superconducting qubits coupled to microwave transmission lines have developed into a versatile platform for solid-state quantum optics experiments [1,2], as well as a promising candidate for quantum computing $[3,4]$. However, compared to optical photodetectors [5-7], detectors for itinerant single-photon microwave pulses are still in their infancy. This prevents microwave implementations of optical protocols that require feedback conditioned on single-photon detection events. For example, linear optical quantum computing with single-photon pulses calls for such feedback [8]. Photodetection and feedback can also act as a quantum eraser [9] of the phase information available in a coherent signal, as we recently discussed in Ref. [10]. Note that, given sufficient averaging, linear amplifiers can substitute for photodetectors in ensembleaveraged experiments $[11,12]$, but the uncertainty principle fundamentally limits the success probability in single-shot experiments.

We focus on thermal photodetectors, i.e., detectors that measure the temperature rise caused by absorbed photons. Thermal detectors have been developed for increasingly long wavelengths in the context of $\mathrm{THz}$ astronomy [13], the record being the detection of single $8 \mu \mathrm{m}$ photons [14]. In the context of quantum thermodynamics [15], thermal detectors have recently been proposed [16] and developed [17-20] as monitorable heat baths.

The other main approach to detecting itinerant microwave photons is to use a qubit that is excited by

Published by the American Physical Society under the terms of the Creative Commons Attribution 3.0 License. Further distribution of this work must maintain attribution to the author(s) and the published article's title, journal citation, and DOI. an incoming photon and then measured [21-30]. Very recently, Ref. [29] reported reaching an efficiency of 0.66 and a bandwidth of roughly $20 \mathrm{MHz}$ using such an approach. Use of qubit-based single-photon transistors as photodetectors has also been proposed [31,32]. If the pulse is carefully shaped, it is also possible to efficiently absorb a photon into a resonator [33-37]. There it could be detected with established techniques for intraresonator photon counting [38,39].

The main advantage of thermal detectors is that they typically present a suitable real input impedance for absorbing photons efficiently over a wide bandwidth and a large dynamic range, in contrast to qubit-based detectors. However, a central problem in the thermal approach is the small temperature rise caused by individual microwave photons. The resulting transient temperature spike is easily overwhelmed by noise added in the readout stage. One potential solution is to use a bistable system as a threshold detector that maps a weak transient input pulse to a longlived metastable state of the detector. This is conceptually similar to, e.g., early experiments on superconducting qubits that used a current-biased superconducting quantum interference device (SQUID) [40]. Conditioned on the initial qubit state, the SQUID either remained in the superconducting state or switched to a long-lived nonzero voltage state.

In this Letter, we show that an electrothermal bistability emerges in the microwave nanobolometer we introduced in Ref. [41] and that it enables high-fidelity threshold detection of $8.4 \mathrm{GHz}$ microwave pulses containing only $200 \times h \times 8.4 \mathrm{GHz} \approx 1.1 \mathrm{zJ}$ of energy. This threshold is more than an order of magnitude improvement over previous thermal detector results [14,42]. The bistability in our detector arises from the fact that the amount of power 


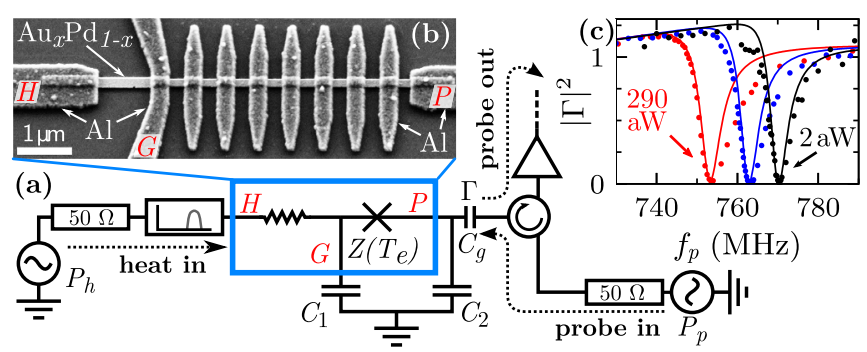

FIG. 1. (a) Simplified diagram of the detector, including (b) a micrograph of the $S-N-S$ junctions formed by a $\mathrm{Au}_{x} \mathrm{Pd}_{1-x}$ nanowire contacted by $\mathrm{Al}$ islands and leads $(H, P$, and $G)$. Here, $Z^{-1}$ is an admittance, $T_{e}$ is the temperature of the electrons in the nanowire, and $\Gamma$ is the probe signal reflection coefficient. The micrograph is from a device nominally identical to the measured one. (c) Reflected fraction of probe power versus probe frequency $f_{p}$ for steady-state heating power $P_{h}$ of $1.9,66$, and $290 \mathrm{aW}$. They are measured at low probe power $P_{p} \ll P_{h}$. The solid curves are fits to the circuit model with a small phenomenological correction term [50]. The heater input is bandpass filtered $(8.41 \pm 0.02 \mathrm{GHz})$.

absorbed from the electrical probe signal used for readout depends on the measured electron temperature itself. Previously, such electrothermal feedback and the associated bifurcation has been studied in the context of kinetic inductance detectors [43-46]. Analogous thermal effects in optics are also known $[47,48]$. The main difference to our device is the relative strength of the electrothermal effect, which in our case leads to strongly nonlinear behavior at attowatt probe powers. Electrothermal feedback is also commonly used in transition edge sensors [13], but typically the feedback is chosen to be negative because that suppresses Johnson noise and leads to fast self-resetting behavior [49].

The central component of our detector (Fig. 1) is a metallic $\mathrm{Au}_{x} \mathrm{Pd}_{1-x}$ nanowire $(x \approx 0.6)$ contacted by three Al leads $(H, P$, and $G)$ and seven $\mathrm{Al}$ islands that are superconducting at millikelvin temperatures [50]. The longest superconductor-normal-metal-superconductor $(S-N-S)$ junction $(H-G)$ provides a resistive load $(36 \Omega)$ for the radiation to be detected [51], while the shorter junctions $(P-G)$ function as a proximity Josephson sensor [52,53]. That is, the shorter junctions provide a temperaturedependent inductance in an effective $L C$ resonator used for readout. Because the inductance increases with electron temperature $T_{e}$ in the nanowire, the resonance frequency shifts down as the heating power $P_{h}$ increases. Therefore the detector transduces changes in $P_{h}$ into changes in the reflection coefficient $\Gamma$ [Fig. 1(c)]. For simplicity, we limit the bandwidth of the heater line using a Lorentzian bandpass filter, but replacing it with a wider band filter should be straightforward.

We first characterize the detector by measuring the $P_{h}$ dependence of the admittance $Z^{-1}$ between $P$ and $G$. To do so, we fit the measured $\Gamma$ to a circuit model in which we
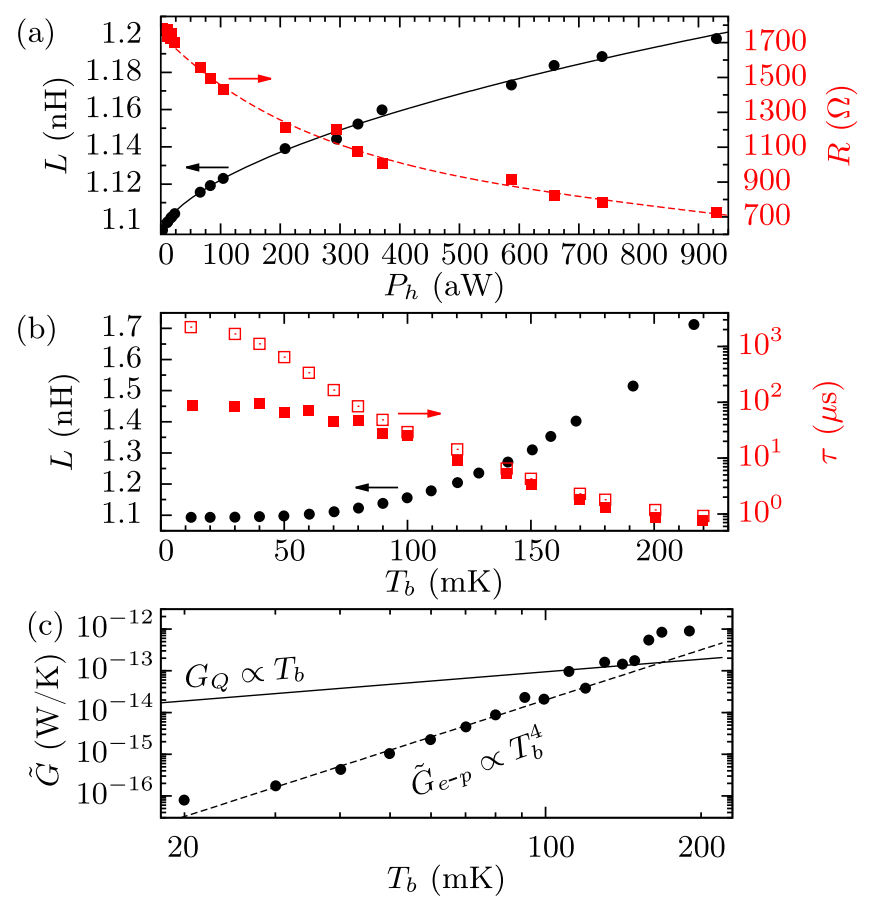

FIG. 2. Linear $\left(P_{p} \ll P_{h}\right)$ response. (a) The effective inductance (circles) and resistance (squares) of the short $S$-N-S junctions as functions of external steady-state heating power $P_{h}$. The bath temperature $T_{b}$ is $12 \mathrm{mK}$. The curves are phenomenological fits that allow mapping a measured reflection coefficient into an equivalent $P_{h}$. (b) The effective inductance (circles) and thermal relaxation time after a short (filled squares) or long (open squares) heating pulse. (c) Measured differential thermal conductance $\tilde{G}$, the expected electron-phonon contribution $\tilde{G}_{e-p}$ (dashed line), and the quantum of thermal conductance $G_{Q}$ (solid line).

parametrize $Z^{-1}$ as $R^{-1}+(i \omega L)^{-1}$, where $\omega / 2 \pi=f_{p}$ is the probe frequency. The circuit model shown in Fig. 1(a) predicts $\Gamma=\left(Z_{L}-Z_{0}\right) /\left(Z_{L}+Z_{0}\right)$, where

$$
Z_{L}=\left(i \omega C_{g}\right)^{-1}+\left\{i \omega C_{2}+\left[\left(i \omega C_{1}\right)^{-1}+Z\left(T_{e}\right)\right]^{-1}\right\}^{-1},
$$

$Z_{0}=50 \Omega, C_{1} \approx 87 \mathrm{pF}, C_{2} \approx 70 \mathrm{pF}$, and $C_{g} \approx 1.5 \mathrm{pF}$. This fits reasonably well to the linear response data shown in Fig. 1(c). However, in order to reproduce the asymmetry in the measured line shape, we add a small frequencydependent correction to the model [50]. Here, linear response refers to the use of a probe power $P_{p}$ low enough to ignore both the electrical and electrothermal nonlinearities, i.e., the nonlinearity of the Josephson inductance as well as the variation of $T_{e}$ as a function of the absorbed probe power $\left(1-|\Gamma|^{2}\right) P_{p}$. We note that the uncertainty in $P_{h}$ is roughly $1 \mathrm{~dB}$ [50], and that dissipation in the capacitors is negligible.

Figure 2(a) shows the extracted linear response $L$ and $R$ for heating powers up to a femtowatt. Figure 2 also shows the bath temperature dependence of $L$, the thermal 
relaxation time $\tau$, and the differential thermal conductance $\tilde{G}=-\partial_{T_{b}} P_{e-b}\left(T_{e}, T_{b}\right)$ [50]. Here, $P_{e-b}\left(T_{e}, T_{b}\right)$ is the heat flow between the electrons in the nanowire and the cryostat phonon bath at temperature $T_{b}$. The measured $\tilde{G}$ is in rough agreement with the prediction for electron-phonon limited thermalization $\tilde{G}_{e-p}=5 \Sigma V_{0} T_{b}^{4}$, where $\Sigma \approx 3 \times 10^{9} \mathrm{~W} / \mathrm{m}^{3} \mathrm{~K}^{5}$ is a material parameter [54] and $V_{0} \approx(240 \mathrm{~nm})^{3}$ is the volume of the part of the nanowire not covered by Al. We can use these results to estimate $C_{e}$ above $100 \mathrm{mK}$, where $T_{e} \approx T_{b}$ and $C_{e} \approx \tau \tilde{G} \approx \gamma V_{0} T_{b}$, with $\gamma V_{0}=8 \mathrm{aJ} / \mathrm{K}^{2}$ [50].

Below $100 \mathrm{mK}$, the relaxation toward the stationary state is faster after a short $(1 \mu \mathrm{s})$ heating pulse than after a long $(\gg \tau)$ heating pulse [50]. Therefore, the simplest thermal model of a single heat capacity $C_{e}$ coupled directly to the bath is not accurate below $100 \mathrm{mK}$. Instead, the second time scale can be phenomenologically explained by an additional heat capacity $C^{\prime} \gg C_{e}$ coupled strongly to $C_{e}$ but weakly to the bath, as compared to $\tilde{G}$. Since $\tilde{G}$ falls far below the quantum of thermal conductance $G_{Q}=$ $\pi^{2} k_{B}^{2} T_{b} / 3 h$ [55] at low temperatures [Fig. 2(c)], even weak residual electromagnetic coupling [56-58] between $C_{e}$ and $C^{\prime}$ would suffice. However, we cannot uniquely determine the microscopic origin of $C^{\prime}$ or the coupling mechanism from the data. Also note that a similar second time scale was observed in Ref. [19].

At high probe powers, the linear-response behavior studied above may be drastically modified by the absorbed probe power. Below we focus on the stationary $T_{e}$ solutions, so we choose to neglect the transient heat flows to $C^{\prime}$ that give rise to the shorter time scale in Fig. 2(b). Similarly, we neglect the contribution of electrical transients to $\Gamma$, as they decay even faster (in $\lesssim 100 \mathrm{~ns}$ ). Under these approximations, $T_{e}$ is the only dynamic variable and evolves according to

$$
\begin{aligned}
C_{e}\left(T_{e}\right) \dot{T}_{e}= & -P_{e-b}\left(T_{e}, T_{b}\right)+P_{x}+P_{h} \\
& +\left(1-\left|\Gamma\left(T_{e}, \omega\right)\right|^{2}\right) P_{p},
\end{aligned}
$$

where $P_{x}$ accounts for the average heat load from uncontrolled sources.

Determining $T_{e}$ from Eq. (1) and the measured $\Gamma$ would require additional assumptions about $P_{e-b}$ and $P_{x}$, as they are not directly measurable. However, we avoid making such assumptions by instead analyzing the increase in the heat flow from the electrons to the thermal bath, as compared to the case $P_{h}=P_{p}=0$. That is, instead of $T_{e}$, we analyze

$$
\Delta\left(T_{e}\right)=P_{e-b}\left(T_{e}, T_{b}\right)-P_{x},
$$

which is monotonic in $T_{e}$. Given this definition, we can rewrite Eq. (1) as

$$
\tau(\Delta) \dot{\Delta}=-\Delta+P_{h}+\left(1-|\Gamma(\Delta, \omega)|^{2}\right) P_{p},
$$

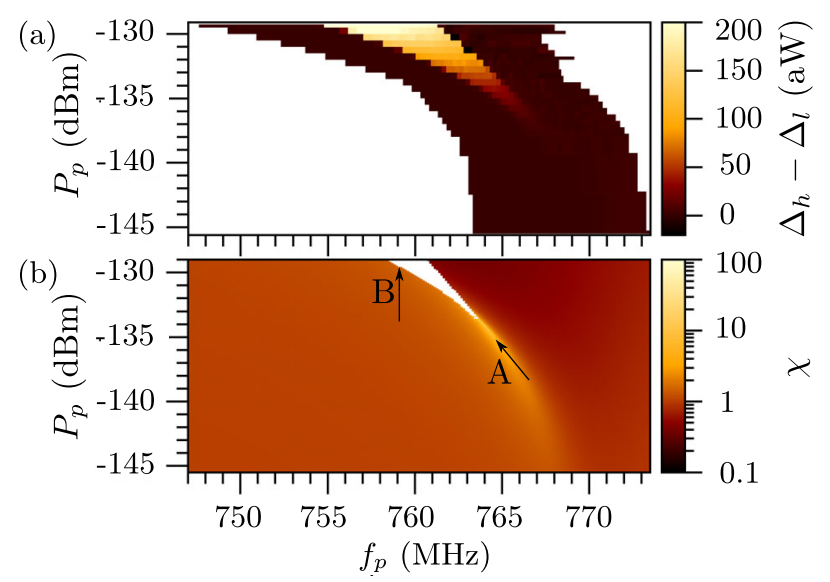

FIG. 3. (a) Bistable parameter regime, as indicated by a nonzero difference $\Delta_{h}-\Delta_{l}$ in the power absorbed from the probe signal in high and low-temperature stationary states. (b) Numerically simulated values of the dimensionless susceptibility to external heating $\chi$ in the single-valued regime. The bistable regime is indicated in white.

where $\tau(\Delta)=C(\Delta) / \partial_{T_{e}} P_{e-b}\left(T_{e}(\Delta), T_{b}\right)$. In contrast to the unknown parameters in Eq. (1), $\Gamma(\Delta, \omega)$ and $\tau(\Delta)$ are directly measurable in linear response. Specifically, we can determine $\Gamma(\Delta, \omega)$ from the data in Fig. 2(a) since $\Delta=P_{h}$ when $P_{p}, \dot{\Delta} \rightarrow 0$. By inverting $\Gamma(\Delta, \omega)$, we can then extract $\Delta$ from the measured $\Gamma$. Also note that, since all parameters in Eq. (3) are determined in linear response, no free parameters remain in the theoretical predictions for the nonlinear case discussed below.

The emergence of bistability is the most dramatic consequence of increasing the probe power. Experimentally, we map out the bistable parameter regime by measuring the difference $\Delta_{h}-\Delta_{l}$ as a function of $f_{p}$ and $P_{p}$ [Fig. 3(a)]. Here, $\Delta_{h}\left(\Delta_{l}\right)$ corresponds to the ensemble-averaged $\Delta$ measured $5 \mathrm{~ms}$ after preparing the system in a high- $\Delta$ (low- $\Delta$ ) initial state. We then identify the region of nonzero $\Delta_{h}-\Delta_{l}$ as the regime where $\Delta$ (and hence $T_{e}$ ) is bistable. This method is approximate mainly because the lifetimes of the metastable states may be short compared to $5 \mathrm{~ms}$.

Figure 3(b) shows the theoretical prediction for the bistable region in white. We generate it by numerically finding the stationary solutions of Eq. (3), with $\Gamma(\Delta, \omega)$ determined from the fits shown in Fig. 2(a) [50]. The qualitative features of the prediction agree well with the experimental results. Quantitatively, the measured bistable regime broadens in frequency faster than the predicted one. This discrepancy is most likely due to imperfect impedance matching of the probe line and the imperfect correspondence between bistability and $\Delta_{h}-\Delta_{l} \neq 0$.

The nonwhite areas in Fig. 3(b) show the prediction for the susceptibility of the stationary-state $\Delta$ to external heating, i.e., $\chi=\partial \Delta /\left.\partial P_{h}\right|_{\dot{\Delta}=0}$. It is a convenient dimensionless way to quantify the importance of the electrothermal nonlinearity. Besides characterizing susceptibility to 
heating, $\chi$ also gives the ratio of the effective thermal time constant to its linear response value. Figure 3(b) shows that both positive $(\chi \gg 1)$ and negative $(\chi \ll 1)$ feedback regimes are accessible by simply choosing different values of $f_{p}$ and $P_{p}$.

There are two distinct ways to operate the device as a detector in the nonlinear regime. Approaching the bistable regime along line $A$ in Fig. 3, the system undergoes a pitchfork bifurcation preceded by a diverging $\chi$. Analogously to the linear amplification of coherent pulses by a Josephson parametric amplifier $[59,60]$, our device could in principle detect heat pulses in a continuous and energy-resolving manner in this regime preceding the bifurcation. However, the focus of this Letter is threshold detection, which uses the imperfect pitchfork bifurcation encountered along line $B$ in Fig. 3 and bears a closer resemblance to the Josephson bifurcation amplifier [61,62].

In the threshold detection mode, we modulate the probe signal amplitude as shown in Fig. 4(a) while keeping the probe frequency fixed at $f_{p}=757 \mathrm{MHz}$. The amplitude modulation pattern first initializes the system to a low- $\Delta$ state, then makes it sensitive to a transition to the high- $\Delta$ state for roughly $t_{s} \approx 4.5 \mu$ s during which the heating pulse is sent, and finally keeps the system in a longlifetime part of the bistable regime for another $7 \mathrm{~ms}$ in order to time average the output signal. During the last stage $P_{p} \approx-131 \mathrm{dBm}$. This is similar to how Josephson bifurcation amplifiers operate [62]. Note, however, that the probe and heater signals do not interfere coherently due to the transduction through electron temperature. That is, at heater frequencies well above $\tau^{-1}$, the output signal is independent of the phase of the heater signal.

The histograms in Figs. 4(b) and 4(c) show that the detector switches reliably to the high-temperature state with a heating pulse energy $E_{\text {pulse }} \gtrsim 1 \mathrm{zJ}$, while it typically remains in the low-temperature state if no heating is applied. The histograms are plotted against $s=\operatorname{Re}\left[e^{1.482 \pi i} \int_{0.8 \mathrm{~ms}}^{6.4 \mathrm{~ms}} d t \Gamma(t) /(5.6 \mathrm{~ms})\right]$, i.e., a projection of the time-averaged reflection coefficient. Few switching events occur during the averaging time, as indicated by the scarcity of points between the two main peaks in the probability density $P(s)$. Instead, the errors arise from spurious early switching events and events where the detector does not switch despite a heating pulse. In particular, for a heat pulse of approximately 200 photons, the readout fidelity is $F=$ 0.56 [Fig. 4(b)]. Here, $F=1-P(s>-0.25 \mid$ no heat pulse $)-$ $P(s \leq-0.25 \mid$ heatpulse $)$. For a heat pulse of 530 photons, $F=0.94$ [Fig. 4(c)]. For 330 photons, $F=0.75$ [50].

The observed pulse energy dependence of $F$ is in agreement with the errors arising mainly from Gaussian fluctuations in the energy of the nanowire electrons. Such fluctuations limit $F$ to $\bar{F}=\operatorname{erf}\left(2^{-3 / 2} E_{\text {pulse }} / \Delta E_{\text {rms }}\right)$, even for ideal instantaneous threshold detection. For rms fluctuations $\Delta E_{\mathrm{rms}}=0.7 \mathrm{zJ}, \bar{F}$ agrees well with the above mentioned values of $F$. This phenomenological $\Delta E_{\mathrm{rms}}$
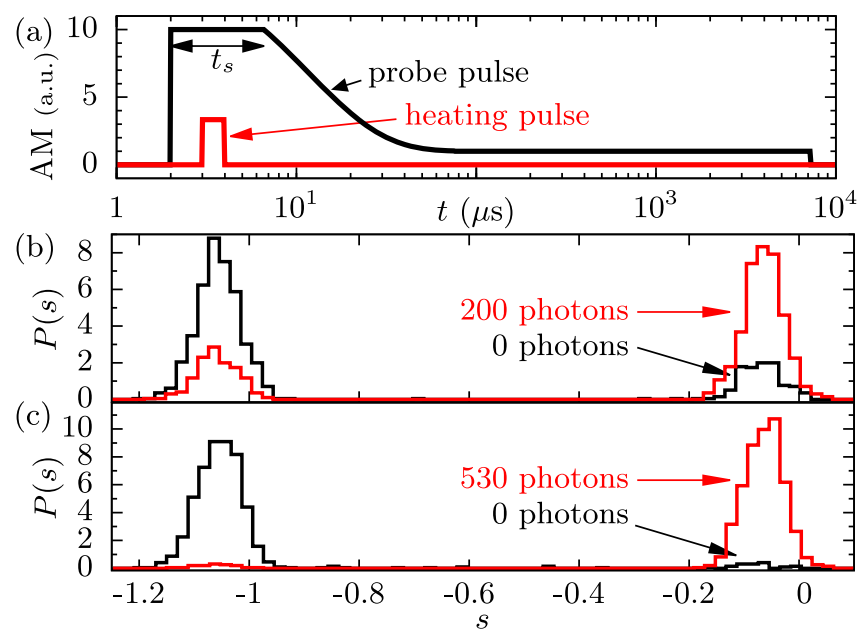

FIG. 4. (a) Amplitude modulation (AM) of the probe pulse used for detecting weak $1 \mu$ s heating pulses (also shown). The carrier frequencies are $757 \mathrm{MHz}$ and $8.4 \mathrm{GHz}$ for the probe and heating pulses, respectively. (b) Normalized histograms of the single-shot measurement outcome $s$ with a heating pulse energy of zero or $200 \times h \times 8.4 \mathrm{GHz} \approx 1.1 \mathrm{zJ}$. The pulses for the two histograms were interleaved in time. (c) Same as (b) but for $3.0 \mathrm{zJ}$ and $t_{s}=2.5 \mu \mathrm{s}$.

should be compared to the thermodynamic fluctuations $\Delta E_{\mathrm{rms}}^{\prime}=\sqrt{k_{B} T_{e}^{2} C_{e}}$ in the absence of electrothermal feedback [63]. For $T_{e}=50 \mathrm{mK}$, we estimate $C_{e} \approx 400 \mathrm{zJ} / \mathrm{K}$, leading to $\Delta E_{\mathrm{rms}}^{\prime} \approx 0.12 \mathrm{zJ} \approx C_{e} \times 0.29 \mathrm{mK}$. This suggests that the thermodynamic fluctuations are a significant, even if not the dominant, fidelity-limiting factor. Note that, although the feedback during the pulse sequence in Fig. 4(a) is strong and positive, all signals are kept off for at least $400 \mathrm{~ms}$ before each probe pulse. Therefore, the fluctuations just before the brief period relevant for switching $\left(t_{s} \ll \tau\right)$ are not affected by the electrothermal feedback.

In conclusion, we have experimentally investigated the electrothermal feedback effect in a microwave photodetector. The results are in agreement with a simple model which we used to highlight that both strong positive and strong negative feedback is available by adjusting the probe power and frequency. We demonstrated that bistability emerges in the limit of extreme positive feedback and that it can be used for efficient threshold detection of weak microwave pulses at the zeptojoule level. This is more than an order of magnitude improvement over previous thermal detector results, and therefore an important step toward thermal detection of individual itinerant microwave photons. To reach the single-photon level, we should further reduce the nanowire volume and possibly replace $\mathrm{Au}_{x} \mathrm{Pd}_{1-x}$ by a material with lower specific heat. This would reduce the time constant as well as the thermodynamic energy fluctuations, which contribute significantly to the achieved fidelities according to our estimate. Furthermore, there seems to be room for technical improvement in shielding 
and filtering, which would bring the observed $\Delta E_{\text {rms }}$ closer to the thermodynamic fluctuations and would, most likely, lead to a lower electron temperature. Finally, a state-of-theart amplifier [64-66] on the probe output should reduce the required averaging time by at least two orders of magnitude [50].

We thank Leif Grönberg for depositing the $\mathrm{Nb}$ used in this work, Ari-Pekka Soikkeli for discussion on modeling the bistability, and Matti Partanen for technical assistance. We also acknowledge the financial support from the Emil Aaltonen Foundation, the European Research Council under Grant No. 278117 ("SINGLEOUT"), the Academy of Finland under Grants No. 265675, No. 251748, No. 284621, No. 135794 , No. 272806, No. 286215, and No. 276528, and the European Metrology Research Programme ("EXL03 MICROPHOTON"). The EMRP is jointly funded by the EMRP participating countries within EURAMET and the European Union. In addition, we acknowledge the provision of facilities by Aalto University at OtaNano-Micronova Nanofabrication Centre.

*joonas.govenius@aalto.fi

[1] A. Blais, R.-S. Huang, A. Wallraff, S. M. Girvin, and R. J. Schoelkopf, Phys. Rev. A 69, 062320 (2004).

[2] A. Wallraff, D. I. Schuster, A. Blais, L. Frunzio, R.-S. Huang, J. Majer, S. Kumar, S. M. Girvin, and R. J. Schoelkopf, Nature (London) 431, 162 (2004).

[3] M. H. Devoret and R. J. Schoelkopf, Science 339, 1169 (2013).

[4] J. Kelly, R. Barends, A. G. Fowler, A. Megrant, E. Jeffrey, T. C. White, D. Sank, J. Y. Mutus, B. Campbell, Y. Chen, Z. Chen, B. Chiaro, A. Dunsworth, I. C. Hoi, C. Neill, P. J. J. O'Malley, C. Quintana, P. Roushan, A. Vainsencher, J. Wenner, A. N. Cleland, and J.M. Martinis, Nature (London) 519, 66 (2015).

[5] A. E. Lita, A. J. Miller, and S. W. Nam, Opt. Express 16, 3032 (2008).

[6] F. Marsili, V. B. Verma, J. A. Stern, S. Harrington, A. E. Lita, T. Gerrits, I. Vayshenker, B. Baek, M. D. Shaw, R. P. Mirin, and S. W. Nam, Nat. Photonics 7, 210 (2013).

[7] M. D. Eisaman, J. Fan, A. Migdall, and S. V. Polyakov, Rev. Sci. Instrum. 82, 071101 (2011).

[8] P. Kok, K. Nemoto, T. C. Ralph, J. P. Dowling, and G. J. Milburn, Rev. Mod. Phys. 79, 135 (2007).

[9] M. Hillery and M. O. Scully, in Quantum Optics, Experimental Gravity, and Measurement Theory, NATO Advanced Science Institutes Series Vol. 94, edited by P. Meystre and M. O. Scully (Plenum Press, New York, 1983) pp. 65-85.

[10] J. Govenius, Y. Matsuzaki, I. G. Savenko, and M. Möttönen, Phys. Rev. A 92, 042305 (2015).

[11] M. P. da Silva, D. Bozyigit, A. Wallraff, and A. Blais, Phys. Rev. A 82, 043804 (2010).

[12] D. Bozyigit, C. Lang, L. Steffen, J. M. Fink, C. Eichler, M. Baur, R. Bianchetti, P. J. Leek, S. Filipp, M. P. da Silva, A. Blais, and A. Wallraff, Nat. Phys. 7, 154 (2011).
[13] B. S. Karasik, A. V. Sergeev, and D. E. Prober, IEEE Trans. Terahertz Sci. Technol. 1, 97 (2011).

[14] B. S. Karasik, S. V. Pereverzev, A. Soibel, D. F. Santavicca, D. E. Prober, D. Olaya, and M. E. Gershenson, Appl. Phys. Lett. 101, 052601 (2012).

[15] J. P. Pekola, Nat. Phys. 11, 118 (2015).

[16] J. P. Pekola, P. Solinas, A. Shnirman, and D. V. Averin, New J. Phys. 15, 115006 (2013).

[17] D. R. Schmidt, C. S. Yung, and A. N. Cleland, Appl. Phys. Lett. 83, 1002 (2003).

[18] D. R. Schmidt, K. W. Lehnert, A. M. Clark, W. D. Duncan, K. D. Irwin, N. Miller, and J. N. Ullom, Appl. Phys. Lett. 86, 053505 (2005).

[19] S. Gasparinetti, K. L. Viisanen, O.-P. Saira, T. Faivre, M. Arzeo, M. Meschke, and J. P. Pekola, Phys. Rev. Applied 3, 014007 (2015).

[20] O. P. Saira, M. Zgirski, K. L. Viisanen, D. S. Golubev, and J. P. Pekola, arXiv:1604.05089.

[21] G. Romero, J. J. García-Ripoll, and E. Solano, Phys. Rev. Lett. 102, 173602 (2009).

[22] Y.-F. Chen, D. Hover, S. Sendelbach, L. Maurer, S. T. Merkel, E. J. Pritchett, F. K. Wilhelm, and R. McDermott, Phys. Rev. Lett. 107, 217401 (2011).

[23] B. Peropadre, G. Romero, G. Johansson, C. M. Wilson, E. Solano, and J. J. García-Ripoll, Phys. Rev. A 84, 063834 (2011).

[24] B. Fan, A. F. Kockum, J. Combes, G. Johansson, I.-C. Hoi, C. M. Wilson, P. Delsing, G. J. Milburn, and T. M. Stace, Phys. Rev. Lett. 110, 053601 (2013).

[25] I.-C. Hoi, A. F. Kockum, T. Palomaki, T. M. Stace, B. Fan, L. Tornberg, S. R. Sathyamoorthy, G. Johansson, P. Delsing, and C. M. Wilson, Phys. Rev. Lett. 111, 053601 (2013).

[26] S. R. Sathyamoorthy, L. Tornberg, A. F. Kockum, B. Q. Baragiola, J. Combes, C. M. Wilson, T. M. Stace, and G. Johansson, Phys. Rev. Lett. 112, 093601 (2014).

[27] B. Fan, G. Johansson, J. Combes, G. J. Milburn, and T. M. Stace, Phys. Rev. B 90, 035132 (2014).

[28] K. Koshino, K. Inomata, Z. Lin, Y. Nakamura, and T. Yamamoto, Phys. Rev. A 91, 043805 (2015).

[29] K. Inomata, Z. Lin, K. Koshino, W. D. Oliver, J.-S. Tsai, T. Yamamoto, and Y. Nakamura, arXiv:1601.05513.

[30] A. Narla, S. Shankar, M. Hatridge, Z. Leghtas, K. M. Sliwa, E. Zalys-Geller, S. O. Mundhada, W. Pfaff, L. Frunzio, R. J. Schoelkopf, and M. H. Devoret, arXiv:1603.03742.

[31] L. Neumeier, M. Leib, and M. J. Hartmann, Phys. Rev. Lett. 111, 063601 (2013).

[32] M. T. Manzoni, F. Reiter, J. M. Taylor, and A. S. Sørensen, Phys. Rev. B 89, 180502 (2014).

[33] J. I. Cirac, P. Zoller, H. J. Kimble, and H. Mabuchi, Phys. Rev. Lett. 78, 3221 (1997).

[34] Y. Yin, Y. Chen, D. Sank, P. J. J. O’Malley, T. C. White, R. Barends, J. Kelly, E. Lucero, M. Mariantoni, A. Megrant, C. Neill, A. Vainsencher, J. Wenner, A. N. Korotkov, A. N. Cleland, and J. M. Martinis, Phys. Rev. Lett. 110, 107001 (2013).

[35] S. J. Srinivasan, N. M. Sundaresan, D. Sadri, Y. Liu, J. M. Gambetta, T. Yu, S. M. Girvin, and A. A. Houck, Phys. Rev. A 89, 033857 (2014).

[36] J. Wenner, Y. Yin, Y. Chen, R. Barends, B. Chiaro, E. Jeffrey, J. Kelly, A. Megrant, J. Y. Mutus, C. Neill, 
P. J. J. O’Malley, P. Roushan, D. Sank, A. Vainsencher, T. C. White, A. N. Korotkov, A. N. Cleland, and J. M. Martinis, Phys. Rev. Lett. 112, 210501 (2014).

[37] M. Pechal, L. Huthmacher, C. Eichler, S. Zeytinoğlu, A. A. Abdumalikov, S. Berger, A. Wallraff, and S. Filipp, Phys. Rev. X 4, 041010 (2014).

[38] S. Gleyzes, S. Kuhr, C. Guerlin, J. Bernu, S. Deléglise, U. Busk Hoff, M. Brune, J.-M. Raimond, and S. Haroche, Nature (London) 446, 297 (2007).

[39] L. Sun, A. Petrenko, Z. Leghtas, B. Vlastakis, G. Kirchmair, K. M. Sliwa, A. Narla, M. Hatridge, S. Shankar, J. Blumoff, L. Frunzio, M. Mirrahimi, M. H. Devoret, and R. J. Schoelkopf, Nature (London) 511, 444 (2014).

[40] I. Chiorescu, Y. Nakamura, C. J. P. M. Harmans, and J. E. Mooij, Science 299, 1869 (2003).

[41] J. Govenius, R. E. Lake, K. Y. Tan, V. Pietilä, J. K. Julin, I. J. Maasilta, P. Virtanen, and M. Möttönen, Phys. Rev. B 90, 064505 (2014).

[42] D. F. Santavicca, B. Reulet, B. S. Karasik, S. V. Pereverzev, D. Olaya, M. E. Gershenson, L. Frunzio, and D. E. Prober, Appl. Phys. Lett. 96, 083505 (2010).

[43] P. J. de Visser, S. Withington, and D. J. Goldie, J. Appl. Phys. 108, 114504 (2010).

[44] S. E. Thompson, S. Withington, D. J. Goldie, and C. N. Thomas, Supercond. Sci. Technol. 26, 095009 (2013).

[45] M. A. Lindeman, J. Appl. Phys. 116, 024506 (2014).

[46] C. N. Thomas, S. Withington, and D. J. Goldie, Supercond. Sci. Technol. 28, 045012 (2015).

[47] V. B. Braginsky, M. L. Gorodetsky, and V. S. Ilchenko, Phys. Lett. A 137, 393 (1989).

[48] A. E. Fomin, M. L. Gorodetsky, I. S. Grudinin, and V. S. Ilchenko, J. Opt. Soc. Am. B 22, 459 (2005).

[49] K. D. Irwin, Appl. Phys. Lett. 66, 1998 (1995).

[50] See Supplemental Material at http://link.aps.org/supplemental/ 10.1103/PhysRevLett.117.030802 for additional single-shot histograms and for details of the experimental setup, the circuit model, the numerical model, and the measurements of $\tilde{G}, \tau$, and $C_{e}$.

[51] M. Nahum and J. M. Martinis, Appl. Phys. Lett. 63, 3075 (1993).

[52] F. Giazotto, T. T. Heikkilä, G. P. Pepe, P. Helistö, A. Luukanen, and J. P. Pekola, Appl. Phys. Lett. 92, 162507 (2008).

[53] J. Voutilainen, M. A. Laakso, and T. T. Heikkilä, J. Appl. Phys. 107, 064508 (2010).

[54] A. V. Timofeev, M. Helle, M. Meschke, M. Möttönen, and J. P. Pekola, Phys. Rev. Lett. 102, 200801 (2009).

[55] J. B. Pendry, J. Phys. A 16, 2161 (1983).

[56] D. R. Schmidt, R. J. Schoelkopf, and A. N. Cleland, Phys. Rev. Lett. 93, 045901 (2004).

[57] M. Meschke, W. Guichard, and J. P. Pekola, Nature (London) 444, 187 (2006).

[58] M. Partanen, K. Y. Tan, J. Govenius, R. E. Lake, M. K. Mäkelä, T. Tanttu, and M. Möttönen, Nat. Phys. 12, 460 (2016).

[59] H. Zimmer, Appl. Phys. Lett. 10, 193 (1967).

[60] M. A. Castellanos-Beltran and K. W. Lehnert, Appl. Phys. Lett. 91, 083509 (2007).

[61] I. Siddiqi, R. Vijay, F. Pierre, C. M. Wilson, M. Metcalfe, C. Rigetti, L. Frunzio, and M. H. Devoret, Phys. Rev. Lett. 93, 207002 (2004).

[62] R. Vijay, M. H. Devoret, and I. Siddiqi, Rev. Sci. Instrum. 80, 111101 (2009).

[63] S. H. Moseley, J. C. Mather, and D. McCammon, J. Appl. Phys. 56, 1257 (1984).

[64] R. Vijay, D. H. Slichter, and I. Siddiqi, Phys. Rev. Lett. 106, 110502 (2011).

[65] M.-O. André, M. Mück, J. Clarke, J. Gail, and C. Heiden, Appl. Phys. Lett. 75, 698 (1999).

[66] C. Macklin, K. O’Brien, D. Hover, M. E. Schwartz, V. Bolkhovsky, X. Zhang, W. D. Oliver, and I. Siddiqi, Science 350, 307 (2015). 